\title{
The locus of the experimental effects in the rapid serial visual presentation (RSVP) task
}

\author{
D. C. MITCHELL \\ University of Exeter, Exeter EX4 4QG, England
}

\begin{abstract}
A central assumption of studies using the rapid serial visual presentation (RSVP) technique is that it taps purely perceptual processes. In an experiment testing this assumption, subjects were presented with seven-word sentences that were either completely intact or had their final three words replaced by three extraneous words. The subjects were significantly less accurate in reporting the first four unchanged words of the sentences in the latter condition. Contrary to assumption, this result indicates that postperceptual factors are critical in the task. It is therefore argued that theoretical conclusions based on the technique should be treated cautiously until the locus of each effect has been established.
\end{abstract}

Experimental investigations of sentence processing have been plagued with methodological problems, many of which derive from the fact that comprehension is a private process with few overt indices. The processing of sentences has been examined experimentally either by requiring subjects to perform an additional task concurrently, such as monitoring the sentence for the presence of a target phoneme (e.g., Foss, 1969), or by examining the process retrospectively, as it is manifested in the way a person recalls, paraphrases, recognizes, or verifies the presented sentence. Neither of these approaches is entirely satisfactory. The monitoring approach imposes an additional task on the subject which may distort the process under examination. Retrospective methods, on the other hand, allow several processing phases to intervene between the initial perception of the sentence and the response, and so it is difficult to distinguish between perceptual and postperceptual effects in this kind of experiment. It follows that any experimental procedure that succeeds in unraveling such effects would be of considerable interest.

Forster and his colleagues (Forster, 1970; Forster \& Ryder, 1971; Holmes \& Forster, 1972) claim to have developed such a technique. In this paradigm, the individual words of a sentence are superimposed visually upon one another in a rapid sequence and, at the end, the subject is required to report as many of the words as possible. The authors maintain that performance in this rapid serial visual presentation (RSVP) task reflects "purely perceptual processes

This work was supported by an S.S.R.C. joint-research grant to the present author and to Dr. D. W. Green. I wish to thank Dr. Green for his help in reworking the first draft of this paper and in contributing to the ideas presented. I am also indebted to Joan Harris for running the experiment. Requests for reprints should be sent to D. C. Mitchell, Department of Psychology, Washington Singer Laboratories, University of Exeter, Exeter, EX4 4QG, England. inasmuch as performance depends primarily on the speed with which $S$ can impose meaningful organization on the input sentence. It should be distinguished (they continue) from other procedures that have little to do with immediate sentence processing" (Forster \& Ryder, 1971, p. 237).

On the basis of the assumption that the RSVP task reflects pure perception, the technique has been used to tackle a number of issues in sentence perception. For example, it has been used to test several of the hypotheses put forward by Fodor, Bever, and Garrett (1974) concerning the perceptual strategies that subjects use to analyze sentences. Thus, Holmes and Forster (1972) presented evidence that sentences with complement verbs are more difficult to process than similar sentences with simple verbs. This is consistent with the verb complexity hypothesis. In another study, Holmes and Watson (1976) obtained results which suggest that the ease with which a sentence can be processed depends in part on the extent to which the deep structure relations are directly revealed in its surface structure. Such a result is compatible with the canonical order hypothesis. Evidence challenging the generality of a third principle, the cue deletion hypothesis, was also obtained in this experiment. Contrary to expectation, the deletion of certain surface structure cues failed to impair recall.

Forster and his colleagues (Forster \& Olbrei, 1973; Forster \& Ryder, 1971) have also used data from the RSVP technique to query the prevailing view that the syntactic processing of a sentence is influenced by its semantic content, and to argue instead for a strictly serial model in which semantic processing follows syntactic processing.

These experiments and other RSVP studies could potentially make an important contribution to the study of sentence perception. However, their actual relevance depends on the validity of the claim that the effects obtained in the RSVP task are solely per- 
ceptual. This claim is based on a number of different assumptions about the nature of visual processing in the RSVP task. First, it is assumed that each new word erases the preceding word so that "any possibility of a cumulative sensory storage of the input is eliminated, and the $S$ is forced to process each word as it occurs" (Forster, 1970, p. 215). Second, it is assumed that variations in performance in different conditions can be attributed to differences in the difficulty subjects have in extracting information from individual frames and that these differences, in turn, depend on the ease with which the word in question can be related to the words already processed (see Forster, 1970, p. 218 and Forster \& Ryder, 1971). Note that, according to this assumption, the processing of an individual word can only be affected by the structure of the sentence if the subject manages to keep strictly up to date with the visual and syntactic analysis of all the preceding words. If he fails to do this, words will be misread and the intended structure will be lost and, in these circumstances, the structure will have no effect on subsequent processing.

The third and perhaps the most important assumption underlying the use of the RSVP technique to investigate psycholinguistic issues is the notion that the effects of sequential structure are restricted exclusively to the extraction process described above. Thus, it is assumed that performance with different materials is not affected differently during storage, retrieval, or reconstruction, or at any other stage of processing after the information has been extracted from the display.

None of these assumptions seem to be plausible in the light of recent research on visual information processing. It seems unlikely that each word is completely erased by its successor. In fact, there is a considerable amount of evidence that when one stimulus is followed by another within $50-100 \mathrm{msec}$ (i.e., the range of intervals typically used in the RSVP task), the first stimulus is integrated with the second and not erased by it (Scheerer, 1973; Schultz \& Eriksen, 1977; Turvey, 1973). More important for the present purposes, there is evidence that visual information can be transferred to a nonmaskable form of visual memory where it may remain available for several seconds (Henderson, 1972; Mitchell, 1972; Phillips \& Baddeley, 1971; see also the review by Coltheart, 1972). In fact, there are strong indications that information of this kind can contribute to performance in an RSVP-type task (Mitchell, 1976). This means that there is no need for each successive word to be identified and assimilated while it remains on display. On the contrary, visual traces of one or more words might survive until the end of the sequence and provide raw data for some kind of reconstruction process before the material is reported.
The second assumption-that the effects of syntactic structure are introduced during the extraction process-also seems unlikely. As pointed out above, the initial readout from the display could only be affected by the structure of the sequence if the lexical, syntactic, and semantic processing of the words proceeds rapidly enough to keep up with the rate at which new stimuli arrive. Experiments employing much simpler stimuli suggest that this is almost certainly impossible. Eriksen and Eriksen (1971) presented subjects with short sequences of stimuli consisting of digits, letters, and arrows, and found that they were not able to process the stimuli accurately at presentation rates in excess of 5 items $/ \mathrm{sec}$. Similar results have been obtained by many other investigators (e.g., Haber \& Nathanson, 1969; Henderson \& Matthews, 1971; Mitchell, 1976). Presumably, the process of identifying words and assimilating them into a syntactic structure is considerably more complex than identifying unrelated digits or letters, in which case it seems unlikely that subjects are able to keep up with processing after the first word or two in the RSVP task. If this is correct, subjects will normally lose track of the structure soon after the beginning of the sequence and, if this hasppens, there is obviously no way in which the structure of the sequence can influence the early stages in the processing of subsequent words. Thus, it is difficult to see how the syntactic and semantic effects that are obtained in RSVP tasks can be explained in terms of differences at the level of the extraction of visual information from the display.

If this argument is accepted, it follows, contrary to the third assumption considered above, that at least some of the structural effects in the RSVP task must be introduced after the extraction process. Perhaps the most plausible suggestion is that subjects extract a few isolated words or word fragments when the materials are first presented and that the structural effects are introduced when they attempt to use this information to reconstruct the original sequence. On this hypothesis, the level of performance might be improved because the subject makes use of linguistic rules to guide his reconstruction of the stimulus sequence, and the variations in performance under different conditions may reflect the fact that the potential benefits of this strategy depend on the degree of structure present in the stimulus sequence.

Forster (1970) has presented some evidence against interpreting RSVP findings in terms of reconstruction effects. In the first of two main arguments against this suggestion, he pointed out that subjects in his study seemed to adhere strictly to their instructions to write down only what they actually saw and not to guess. For example, there were occasions when a space was left in the report form even when the missing word was highly predictable (Footnote 2, 
p. 221). The second line of argument was based on the results of an experiment conducted specifically to test the reconstruction hypothesis. Subjects were required to decipher blurred typewritten copies of sentences similar to those used in the RSVP task. It was argued that, if sentences of one structure were more redundant than those of another, then performance on this task should reflect the difference. In fact, the crucial difference was small and nonsignificant, and so Forster concluded that the corresponding finding in the RSVP experiment could not be attributed to reconstruction.

Neither of these arguments is particularly persuasive. The finding that predictable words were sometimes excluded from report merely indicates that some subjects refrained from guessing on some trials and does not (and, in fact, cannot) establish that this strategy was adopted universally. It is quite likely that there were other instances that could be interpreted as evidence in favor of guessing and reconstruction. The second argument is also unconvincing, since, as Forster himself observed, the reconstruction experiment was based on a failure to reject the null hypothesis. Moreover, since fewer subjects were used than in the main experiment, the differences between the conditions might have failed to materialize because the experiment was insensitive and not because reconstruction has an insignificant effect on performance. In fact, results obtained in subsequent studies using the same method appear to indicate that Forster's initial rejection of the reconstruction hypothesis was unwarranted. Forster and Olbrei (1973) showed that one of the variables that influence performance in the RSVP task (sentence plausibility) also has a strong effect in the reconstruction task, which suggests that reconstruction may contribute to performance in the RSVP version of the task.

The preceding discussion suggests that the structural differences in the RSVP task are not likely to be introduced during the extraction of information from the visual display. In fact, despite Forster's arguments to the contrary, the most likely interpretation of the data seems to be that the effects occur during some kind of reconstruction process. However, the evidence against a "purely perceptual" interpretation of RSVP data is somewhat indirect and much of it is based on experiments that bear little resemblance to the RSVP task or experiments employing markedly different kinds of stimulus materials.

The present experiment was, therefore, conducted to examine the issue more directly, using strings of words and sentences similar to those employed by Forster and his colleagues. In this study, the accuracy of report of normal seven-word sentences is compared with that of sentences in which the last three words are replaced by three extraneous words. According to the "purely perceptual" hypothesis, structural differences should only influence the extraction process, and since the first four words should have been read out before any differences between the conditions became apparent, there is no reason to expect any difference in the ease with which these words are assimilated. Thus, it would be expected that any differences between the conditions would be confined to the last three positions. On the other hand, if the effects are not "purely perceptual" (i.e., if they are introduced during storage, retrieval, or reconstruction), performance could easily be affected in all seven positions. For example, on the reconstruction hypothesis considered above, the extraneous words may yield misleading information which could hamper the subjects' efforts to generate an acceptable reconstruction of the sentence as a whole. (Also, on this hypothesis, subjects might pause longer in the extraneous-words condition before they started to report the sentences. Measures of response latency were therefore recorded during the experiment in order to test this possibility.)

\section{METHOD}

\section{Sentences}

The sentences were seven words in length and were based on the "plausible" sentences used by Forster and Ryder (1971). There were 24 sentences consisting of only one clause (simple sentences) and 24 two-clause sentences (complex sentences). Eight sentences of each kind were taken from the materials used by Forster and Ryder. Two new sentences were modeled on each of these original sentences. In each case, the surface structure was identical to that of the model sentence and the length of each word was also approximately the same.

In addition to the normal sentences, there were 48 partially scrambled sentences. In each of these, the first four words were identical to the first four words of one of the normal sentences. However, the last three words were different. They were sampled, without replacement, from one of the two pools of 72 words that made up the endings of the normal sentences. In the case of the simple sentences, the endings were drawn from the pool of endings for simple sentences and, likewise, two clause endings came from the complex sentences. The only constraint in the selection of the three final words was that the new seven-word sequence should not constitute an acceptable English sentence. This method of constructing the scrambled sentences means, of course, that in the pool of materials as a whole, the words are identical to those in the set of normal sentences.

In addition to the experimental sentences, there were a further eight simple sentences and eight complex sentences. These were all normal sentences (i.e., not scrambled) and were intended to give the subjects the impression that the sentences were predominantly normal. They were not included in the data analysis.

\section{Procedure}

The word sequences were presented on a DEC VR12 pointplot display with a P31 phosphor. The presentation was controlled by a PDP-12 computer. The words were superimposed on one another at the rate of 20 words/sec, and there was no blank interval between the successive words. The materials were displayed in uppercase letters $(.6 \mathrm{~cm}$ high and between .3 and $.5 \mathrm{~cm}$ wide) in the center of the screen. The middle letter of each word 
(or one of the two middle letters) always appeared in the same location. The visual angle subtended at the eye ranged from $.5^{\circ}$ to approximately $5^{\circ}$, depending on the length of the word. The average luminance was $13.5 \mathrm{~mL}$ and there was a background luminance of approximately $.4 \mathrm{~mL}$ due to reflections from the CRT screen.

At the beginning of each trial, there was a continuous display consisting of a dense pattern of random dots occupying eight letter spaces. This was intended to mark the position on the screen where the stimuli would appear. The trial was initiated when the subject pressed a "start" button. After this, the pattern remained on display for a further $500 \mathrm{msec}$ before the first word appeared. At the end of the sequence, two vertical lines were displayed continuously until the subject pressed a second button labeled RTW (for "ready to write"). After this response, the dot pattern reappeared for the start of the next trial. There was a short pause after every 10 trials while the computer compiled the material for the next 10 sentences.

The subjects were instructed to write down the words in order as accurately as possible. They recorded their responses in an answer booklet with seven spaces on a separate page for each trial, and they were told to leave spaces in the appropriate places when they were unable to remember the words. They were told that they should try to decide what they were going to write before they began, and they were instructed to press the "ready to write" button before actually writing the sentence. It was explained that most of the sequences would be perfectly natural English sentences. The instructions concerning guessing were almost identical to those used by Forster and Ryder (1971): "It is important not to guess unless you are reasonably sure that you know what the sentence was. To guard against this, a few sentences will be slightly jumbled, and your performance on these will be checked for guessing."

There was a practice block of 10 trials before the main experiment to familiarize subjects with the experimental technique. In this practice session, the first few trials were presented much more slowly than in the main experiment.

During the experiment, each subject saw each sentence either in its normal or in its scrambled form. There were 24 normal sentences (12 simple and 12 complex) and 24 scrambled (again 12 of each). Eight different randomizations were used to ensure that over the whole experiment the four different types of material appeared equally often in each position in the sequence of trials and to assure that half the subjects each saw a particular sentence in its two alternative forms. The experimental session lasted approximately $45 \mathrm{~min}$.

\section{Subjects}

The subjects were 12 male and 12 female unpaid undergraduate volunteers drawn from the subject pool at Exeter University.

\section{RESULTS}

The accuracy of recall was determined for each subject and for each trial. Obvious spelling errors were ignored in the analysis, and each item was scored for its correct position in the sequence. The mean percentage of items correctly recalled was computed for the beginning of each sequence (Positions 1-4) and the end (Positions 5-7) both for one- and for two-clause sentences and for normal and scrambled sentences. The position scores in each condition are shown in the top half of Table 1.

It is necessary to check that the present experiment was comparable with previous RSVP studies. With this purpose in mind, it was originally designed as a
Table 1

The Percentage of Words Correctly Reported From the Beginnings (Positions 14) and Ends (Positions 5-7) of One- and Two-Clause Sentence for Normal and Scrambled Sentences

\begin{tabular}{|c|c|c|c|c|}
\hline \multirow{4}{*}{$\begin{array}{l}\text { Scoring System and } \\
\text { Sentence Type }\end{array}$} & \multicolumn{4}{|c|}{ Sentence Complexity } \\
\hline & \multicolumn{2}{|c|}{ One Clause } & \multicolumn{2}{|c|}{ Two Clauses } \\
\hline & \multicolumn{4}{|c|}{ Positions } \\
\hline & 14 & $5-7$ & $1-4$ & $5-7$ \\
\hline $\begin{array}{l}\text { Position Scoring } \\
\text { Normal Sentences } \\
\text { Scrambled Sentences }\end{array}$ & $\begin{array}{l}73.00 \\
53.75\end{array}$ & $\begin{array}{l}62.56 \\
32.51\end{array}$ & $\begin{array}{l}71.96 \\
64.74\end{array}$ & $\begin{array}{l}64.19 \\
29.91\end{array}$ \\
\hline $\begin{array}{l}\text { Order Scoring* } \\
\text { Normal Sentences } \\
\text { Scrambled Sentences }\end{array}$ & $\begin{array}{l}83.58 \\
73.56 \\
\end{array}$ & $\begin{array}{l}74.56 \\
47.69 \\
\end{array}$ & $\begin{array}{l}77.23 \\
73.81 \\
\end{array}$ & $\begin{array}{l}73.53 \\
42.33 \\
\end{array}$ \\
\hline
\end{tabular}

*The second scoring system was the same as that used by Forster (1970), p. 216.

partial replication of the experiment reported by Forster and Ryder (1971). The results shown in Table 1 reveal that the level of performance for normal sentences was higher than that in the previous study. This may be attributed to factors such as background illumination, the visual angle presented by the stimuli, and various other experimental differences. Whatever the cause, it does not seem to be likely to be significant for the interpretation of the remaining results of the experiment.

The report data were entered into a repeated measures analysis of variance for sentence type (normal vs. partially scrambled) by complexity (oneor two-clause sentences) by subjects (or materials). Both subjects and materials were treated as random effects (Clark, 1973). The main effect of sentence type was highly significant $\left[\min F^{\prime}(1,64)=20.91\right.$, $\mathrm{p}<.01$, replicating Forster's (1970) finding that scrambled strings are recalled more poorly than sentences. However, contrary to expectations, neither the complexity effect nor its interaction with sentence type were significant $\left(F_{1}, F_{2}<1\right.$, in each case). This means that the experiment apparently failed to replicate the complexity finding reported by Forster (1970) and Forster and Ryder (1971). It is possible that the results were distorted by the data from the scrambled sentences. The normal data were therefore reanalyzed alone, but there was still no evidence of a complexity effect (both $F_{1}$ and $F_{2}<1$ ).

The apparent failure to replicate the complexity effect may be attributable to the use of a scoring procedure that differed from that employed by Forster and his colleagues. In each of the earlier studies, words were scored correct if they were recalled in the correct order, wherever they appeared in the report sequence (see Forster, 1970, p. 216). Accordingly, the present data were rescored, and it was found that, for normal sentences, $79.65 \%$ of words were 
correctly reported in the one-clause condition and $76.27 \%$ in the two-clause condition (see Table 1 , lower half). Although this difference is only about half as big as that obtained by Forster and Ryder, it is reliable enough to generalize to new subjects $\left[F_{1}(1,23)=7.51, p<.025\right]$, and so it can be concluded that the complexity effect has been replicated for the specific materials and experimental conditions used in the present study.

The replications of earlier findings suggest that the present experiment is comparable with other RSVP studies. It is therefore possible to turn to the main issue of interest: whether the level of performance in Positions 1-4 is affected by the nature of the subsequent material in the stimulus sequence. The data for the first four words were submitted to a repeated measures analysis of variance for Sentence Type by Complexity by Subjects (or Materials). There was a significant reduction in performance for sentences with scrambled endings $\left[\min F^{\prime}(1,68)=7.126\right.$, $\mathrm{p}<.01$ ], using position scores and $\min \mathrm{F}^{\prime}(1,62)=$ $10.18, \mathrm{p}<.01$, using Forster's scoring procedure). Neither the complexity effect nor its interaction with sentence type were significant $\left(\min F^{\prime}<1.5\right.$, in each case).

The only remaining result of the experiment concerned the response preparation time, that is, the interval between the end of the stimulus sequence and the time when the subject pressed the "ready to write" button. Median latencies were submitted to an analysis of variance with factors for sentence type, complexity, subjects, and sex. The only significant effect was the difference between preparation time for normal sentences $(2.37 \mathrm{sec})$ and scrambled sentences $(2.53 \mathrm{sec})\left[\mathrm{F}_{1}(1,22)=4.61, \mathrm{p}<.05\right]$, and this effect was not sufficiently reliable to generalize to new materials. This lack of sensitivity may have occurred partly because subjects disobeyed the instruction to decide that they were going to write before responding. For example, on some occasions they made a few words, crossed them out, and started again in a different way. This suggests that they were reorganizing the material while they were writing. It is clear that response latency was inaccurate and unsatisfactory as a measure of response preparation time, and it is difficult to draw any conclusions from these data. Nevertheless, the fact that the latencies were between 2 and $3 \mathrm{sec}$ in duration for both novel and unscrambled sentences suggests that subjects were engaged in some nontrivial processing immediately after the end of the sequence.

\section{DISCUSSION}

The results show clearly that, in the present experiment, the level of performance for the early words was influenced by the nature of the materials that appeared later in the stimulus sequence. This effect cannot be explained in terms of differences that arise during the extraction processes, since these processes should have been completed before the last three words arrived. Moreover, the materials in the first few positions were counterbalanced over the experiment, and so there is no reason why there should have been any systematic differences in the initial registration of these words. It follows that performance must have been influenced at some point after the information had been extracted from the visual stimulus.

Before considering alternative interpretations of the effect, it is perhaps worth discussing the generality of the present findings. It may be that postextraction effects only occur under the conditions of the present study and would disappear if the procedural details were changed. In fact, this seems unlikely, since some of the results of the present experiment indicate that it is comparable with earlier RSVP studies. In particular, the gross difference between normal and scrambled sentences reported by Forster (1970) was replicated, and so was the more delicate complexity effect reported by Forster and Ryder (1971). In any case, there appears to be independent evidence that there are retrospective effects on the level of performance in the RSVP task. Forster (Note 1) conducted an experiment in which subjects were presented with sentences such as:

(1) The old man was spitting thanking on the floor.

(2) The old man was spitting thanking the young boys.

and were instructed to report exactly what they saw. It was found that performance was influenced by the nature of the sentence endings. That is, the subjects who saw Sentence 1 were more likely to report "spitting" than "thanking," while the reverse was the case for Sentence 2. This implies that subjects' performances in the middle of the sentence were influenced after they had processed the last three words. These results, together with the results of the present experiment, are consistent with the notion that performance in RSVP tasks is influenced during processes that occur after information has been extracted from the stimulus display.

This strongly suggests that postperceptual effects occur in the RSVP task. However, it could conceivably be argued that the postextraction processes are "perceptual" even though they do not operate on the stimuli themselves. More specifically, they may be perceptual processes that lag behind at the rate at which new stimuli arrive and use stored representations of these stimuli as their raw material. It would be necessary to make a number of additional assumptions about such "lagging perceptual processes" to 
explain the retroactive interference effect demonstrated in the present experiment. In particular, it is necessary to assume that certain aspects of the processing of the last three words are carried out before the analysis of the first four words has been completed. If this were not the case (i.e., if the processing of the early material were always completed before the work on the later material was started), then there would be no way in which the processing of the later words could influence the perceptual analysis of the earlier material.

Presumably, the most likely version of the "lagging perceptual process"' hypothesis is that difficulties encountered at an early stage in the processing of the last three words interfere with a later stage in the analysis of the first part of the sentence. If we suppose that the extraction of visual features from a display takes less time than identifying a word, and that word recognition, in turn, is completed before the parsing and semantic processing can occur, then it can be assumed that the first two processes are more likely to be completed early enough to interfere with the processing of the first four words than the parsing and semantic processes are. (In any case, it is doubtful whether the parsing of the later words could proceed until the parsing of the earlier words had been completed, because the way in which the string of words is parsed normally depends on the context in which they appear.)

Thus, the most likely "perceptual" explanation of the retroactive interference effect is that some aspect of the early processing of the last three words is responsible for the interference. However, in order to account for the data, this interference must be greater in the scrambled condition than in the normal condition, and it is difficult to see why this should be so. Superficially, the explanation may seem obvious. The fifth and later words appeared in an inappropriate context, and so it is possible that they were more difficult to identify than the corresponding words in the normal condition. If this were the case, the interference with the earlier perceptual processing would presumably be greater in this condition, and we would have a reasonable way of interpreting the results in terms of perceptual effects. However, this explanation is completely unsatisfactory, since there could only be a contextual effect in the recognition of the fifth and later words if the first part of the sentence had already been perceived at this point. But this defeats the whole purpose of the exercise, since, if the first part of the sentence has already been perceived, it is obvious that any interference effects must be postperceptual, which is precisely what the explanation set out to avoid. It follows that, if the fifth and later words are difficult to process in the scrambled condition, it must be for some reason other than the fact that they failed to fit in with the preceding context. In other words, the difficulty could only be associated with the properties of the words themselves (e.g., their lengths, frequencies, etc.). However, over the experiment as a whole, the words in the scrambled condition were exactly the same as those in the normal condition, and so it is difficult to see how there could have been any differences of this kind.

The foregoing discussion suggests that the present results cannot be explained convincingly on the basis of differences at the level of "purely perceptual processing," even when these processes lag behind the input. Thus, the experiment demonstrates that performance in the task can be influenced by nonperceptual factors. The present data do not enable us to specify whether the major effects occur during storage or during the retrieval or reconstruction of the sentence. However, the main point is that experimental effects in the RSVP task are not necessarily perceptual.

It follows from this that RSVP studies do not necessarily tell us anything about the perception of sentences. If positive results are obtained in such studies, the only conclusion that can be drawn is that there is some effect either during perception or during storage, retrieval, or reconstruction of the sentence. Obviously, this is of limited value if the investigator is primarily interested in perceptual processing.

It seems likely that at least a few of the results of the RSVP experiment have been wrongly attributed to perceptual effects. For example, consider the finding that sentences containing transitive verbs are reported more accurately than sentences with complement verbs (Holmes \& Forster, 1972). This result was interpreted as evidence in favor of the verb complexity hypothesis put forward by Fodor et al. (1974). However, recent studies using direct measures of immediate processing have thrown doubt on this conclusion. Hakes (1971), using a phoneme monitoring task, found no differences in response time to a target phoneme following transitive and complement verbs. Also, Mitchell and Green (1978), using continuous text, found no differences in the time needed to comprehend three-word displays that contained the direct object of an immediately preceding transitive or complement verb. These results suggest that the verb complexity effect is unlikely to be a perceptual phenomenon. If this is correct, it follows that the conclusions originally derived from the RSVP experiment were misleading.

In summary, the effects that occur in studies employing the RSVP technique could potentially arise during the storage, retrieval, or reconstruction of the stimulus material as well as (or instead of) during the perception of the sentence. Proponents of the technique have presented arguments that the task 
reflects "purely perceptual processes." However, these arguments were considered at length in the introduction and were found to be unconvincing. An experiment was carried out to demonstrate empirically that performance in the RSVP task can be influenced by nonperceptual effects, and the results confirmed that this was the case. It is concluded that the results of RSVP studies are difficult to interpret and that the conclusions drawn from such studies should be treated with caution until the locus of the effect has been properly established.

\section{REFERENCE NOTE}

1. Forster, K. I. The role of semantic hypotheses in sentence processing. Colloques Internationaux du C.N.R.S. No. 206Problèmes actuels en psycholinguistique.

\section{REFERENCES}

Clark, H. H. The language-as-a-fixed-effect fallacy. A critique of language statistics in psychological research. Journal of Verbal Learning and Verbal Behavior, 1973, 12, 335-359.

ColthearT, M. Visual information-processing. In P. C. Dodwell (Ed.), New horizons in psychology (2). Harmondsworth, Middlesex: Penguin Books, 1972.

ERIKSEN, C. W., \& ERIKsen, B. A. Visual perceptual processing rates and backward and forward masking. Journal of Experimental Psychology, 1971, 89, 306-313.

Fodor, J. A., BEVER, T. G., \& GARRETT, M. The psychology of language. New York: McGraw-Hill, 1974.

Fonster, K. I. Visual perception of rapidly presented word sequences of varying complexity. Perception \& Psychophysics, $1970,8,215-221$.

Forster, K. I., \& Olb REI, I. Semantic heuristics and syntactic analysis. Cognition, 1973, 2, 319-347.

Forster, K. I., \& Rrder, L. A. Perceiving the structure and meaning of sentences. Journal of Verbal Learning and Verbal Behavior, 1971, 10, 285-296.
Foss, D. J. Decision processes during sentence comprehension: Effects of lexical item difficulty and position upon decision times. Journal of Verbal Learning and Verbal Behavior, 1969, 8, 457-482.

Haber, R. N., \& Nathanson, L. S. Processing of sequentially presented letters. Perception \& Psychophysics, 1969, 5, 359-361.

HAKEs, D. T. Does verb structure affect comprehension? Perception \& Psychophysics, 1971, 10, 229-232.

Henderson, L. Visual and verbal codes: Spatial information survives the icon. Quarterly Joumal of Experimental Psychology, 1972, 24, 439-447.

Henderson, L., \& Matrhews, M. L. Perception and memory loss of item and order information in short-term memory. Perception \& Psychophysics, 1971, 9, 231-233.

Holmes, V. M., \& Forster, K. I. Perceptual complexity and underlying sentence structure. Journal of Verbal Learning and Verbal Behavior, 1972, 11, 148-156.

Holmes, V. M., \& WATson, I. J. The role of surface order and surface deletion in sentence perception. Quarterly Journal of Experimental Psychology, 1976, 28, 155-165.

Mitchels, D. C. Short-term visual memory and pattern masking. Quarterly Joumal of Experimental Psychology, 1972, 24, 394-405.

MrtChell, D. C. Buffer storage modality and identification time in tachistoscopic recognition. Quarterly Joumal of Experimental Psychology, 1976, 28, 325-337.

Mrtchell, D. C., \& GREen, D. W. The effects of context and content on immediate processing in reading. Quarterly Journal of Experimental Psychology, 1978, 30, 609-636.

Phillips, W. A., \& Baddeley, A. D. Reaction time and visual short-term memory. Psychonomic Science, 1971, 22, 73-74.

SCHEERER, E. Integration, interruption and processing rate in visual backward making: II. An experimental test. Psychologische Forschung, 1973, 36, 95-115.

Schultz, D. W., \& ERIKsen, C. W. Do noise masks terminate target processing? Memory \& Cognition, 1977, 5, 90-96.

TuRVEY, M. T. On peripheral and central processes in vision. Psychological Review, 1973, 80, 1-52.

(Received for publishing June 7, 1978; revision accepted November 8,1978 .) 